\title{
Guillain-Barre Syndrome in 2016: The Centenary Advances
}

\author{
Bhawna Sharma and Madhuparna Paul
}

\section{Bhawna Sharma, Madhuparna Paul}

Dept. of Neurology, SMS Medical College and hospital, Jaipur, Rajasthan

\section{Correspondence}

Dr.Bhawna Sharma

"Chandraratan" House no. 381, Laxmanpath, Vivek vihar, New Sanganer road, Sodala, Jaipur, Rajasthan. Mobile-094140 75120; 09829219314 Email: sharmadrbhawna@gmail.com; dr.madhuparna@gmail.com

\section{History}

- Submission Date: 03-09-15;

- Review completed: 18-06-16;

- Accepted Date: 22-06-16.

DOI : 10.5530/ijmedph.2016.3.2

Article Available online

http://www.ijmedph.org/v6/i3

\section{Copyright}

(C) 2016 Phcog.Net. This is an open-access article distributed under the terms of the Creative Commons Attribution 4.0 International license.
Guillain-Barre syndrome (GBS) is the most common and severe acute paralytic polyradiculoneuropathy. Since its initial description by Guillain, Barre and Strohl in the year $1916^{1}$ there has been a huge expansion in the knowledge of this potentially treatable disorder. 2016 marks the centenary year of GBS. It was conventionally described as an acute onset ascending pure motor demyelinating illness with areflexia. It has an annual incidence of 1/1000,000 across several studies. ${ }^{1}$ It can occur at any age with a slight male preponderance ${ }^{1}$ and with seasonal variations. ${ }^{4}$ However with ever growing knowledge in last 100 years the clinical spectrum under this umbrella has also expanded and several subtypes based on histopathology and neurophysiology have emerged. The various forms of GBS are Acute Inflammatory Demyelinating Polyneuropathy (AIDP), Acute Motor Axonal Neuropathy (AMAN), Acute Motor and Sensory Axonal Neuropathy (AMSAN), Miller Fisher Syndrome(MFS). ${ }^{2}$ AIDP is the more common in the western world while AMAN is more common in Asian subcontienent (in Japan and China). ${ }^{1}$ Other variants like pandysautonomia, pure ataxic GBS, pharyngeal- cervical-brachial GBS, bibrachial onset GBS and isolated bulbar palsy ${ }^{2}$ have also been described. Few cases may have retained reflexes, positive babinski sign, papilloedema ${ }^{1}$ and transient bladder involvement.

Two third of cases are preceeded by antecedent infections either bacterial or viral .Campylobacter jejuni (C. jejuni), Epstein Bar virus (EBV) or Cytomegalovirus (CMV), ${ }^{2}$ Hepatitis E, ${ }^{8}$ Mycoplasma $^{1}$ and recently Zika virus ${ }^{4}$ are responsible organisms. ${ }^{3}$ Immunizations (swine flu or rabies vaccine), insect bites, pregnancy, surgery, cancer, autoimmune diseases, spinal anaesthesia may also act as trigger. ${ }^{2}$ It is an immune mediated process and the basic pathogenesis involves autoimmunity and complement activation. HLA subtypes ${ }^{2}$ probably also play role. Molecular mimicry with cross reactivity between peripheral nerve ganglioside and antibody against lipooligosaccharides of infectious agents act as a trigger to initiate aberrant immune reactions causing destruction of peripheral nerves. ${ }^{4}$ About half of the cases has autoantibodies against peripheral nerve gangliosides. The syndrome evolution is quite characterstic with onset within 7-21 days of an acute respiratory tract infection or gastroenteritis with ascending areflexic paralysis with or without subjective sensory symptoms.
The disease reaches its peak within 2-4 weeks followed by a plateau phase and then recovery. The syndrome often mimics other conditions like Hypokalaemic periodic paralysis, Acute Demyelinating Encephalomyelitis, Toxic neuropathy, Acute transverse myelitis in shock stage, Cauda equine syndrome, HIV radiculitis and Critical illness neuropathy. ${ }^{4}$ Acute onset CIDP and Treatment Related Fluctuations (TRF) also need differentiation and appropriate management. Diagnostic criteria laid by Asbury and colleagues is still of utmost important. ${ }^{4}$ CSF cell count is increased with cell count often less than $50 / \mathrm{cmm},{ }^{5}$ Seen after first week of infection and Nerve Conduction Study shows demyelinating or axonal abnormality based on the subtypes. Both of these are supportive tools for correct diagnosis. Recently Brighton criteria ${ }^{5}$ have been used for diagnostic purpose.

There is various grading scale for prognostication of GBS based on which therapeutic strategies are planned. Some commonly used are Medical Research Council (MRC) score, the Erasmus GBS outcome score (EGOS), ${ }^{6}$ Erasmus GBS respiratory insufficiency score (EGRIS) and Hughes et al grading system, ${ }^{7}$ of which MRC grading and Hughes grading are most commonly used. Patients should be carefully monitored for respiratory involvement which occurs suddenly. About $25-30 \%$ of Patients are subjected to mechanical ventilation. Dysautonomia, in milder form seen in three quarter of patients, ${ }^{1}$ is another dreadful complication. Hughes functional grading scale for GBS is used to assess the functional disability of the patients. Grade 0 normal functional state, Grade 1 able to run with minor signs and symptoms, Grade 2 able to walk $5 \mathrm{~m}$ independently, Grade 3 able to walk $5 \mathrm{~m}$ with aid, Grade 4 bed or chair bound and Grade 5 require assisted ventilation. ${ }^{7}$ Grade 5 patients carry poor prognosis. Poor prognosis is also seen with older age, rapid progression of disease, severe disease indicated by MRC score, preceeding diarrhoea, positive serology for C.jejuni and $C M V,{ }^{6}$ early cranial nerve involvement.

Being an autoimmune disease immunotherapy is the mainstay of treatment. Intravenous Immunoglobulin (IvIg), ${ }^{6}$ Plasma Exchange $(\mathrm{PE})^{6}$ and Intravenous pulse Methyprednisolone (Iv MPS) ${ }^{2}$ have been tried. IvIg $(0.4 \mathrm{gm} / \mathrm{kg}$ over 5 days $)$ is the gold standard treatment in view of its ease of administration and efficacy. The only drawback is its cost. PE is 
cumbersome procedure in many setups and has various adverse effects. Though IvMPS has been refuted by many researchers, but in author's experience in developing countries like India, it may be a good therapeutic option. Newer drugs like Eculizumab, ${ }^{4}$ a humanized monoclonal antibody that binds C5 complement component is still underway. About 10\% patients who received IvIg may show deterioration in first ${ }^{8}$ weeks (Treatment Related Fluctuations) and they often require repeat IvIg. Thirty five percent patient recovers completely, $35 \%$ has minimal residual motor deficits, $30 \%$ has moderate to severe residual paresis. Mortality ranges from $5-10 \%$ (1) in most studies, the cause being respiratory involvement.

\section{ACKNOWLEDGEMENT}

NIL.

\section{CONFLICT OF INTEREST}

There is no conflict of interest.

\section{REFERENCES}

1. Winer JB. An update in Guillin-Barre syndrome. Queen Elizabeth Hospital. Autoimmune Diseases. 2014. http://dx.doi.org/10.1155/2014/793024. PMid:24511391
PMCid:PMC3910670.

2. Zhong Min, Cai Fang-Chang. Current perspectives on Guillin-Barre syndrome. Department of Neurology children Hospital Chongquing Medical University. World J Pedialr. 2007;3(3):187-94.

3. Winer J B, Hughes R A C, Anderson M J et al. A prospective study of acute idiopathic Neuropathy, II Antecedent events. Department of Neurology St Mary's Hospitals. Journal of Neurology, Neurosurgery and Psychiatry.1988;51(5):613-8. http://dx.doi.org/10.1136/jnnp.51.5.619;http://dx.doi.org/10.1136/jnnp.51.5.613; PMid:3404161 PMCid:PMC1033063.

4. Willson Hugh J, Jacobs Bart C, VenDoorn Peter A. Guillin-Barre syndrome. Immunity and Inflammation College of Medical, Vetenary and Life Science University of Glassgow. Seminar. 2005.

5. Fokke Christiaan, Berg Ven den Bianca, Drenthen Judith et al. Diagnosis of Guillin-Barre syndrome and validation of brighton criteria. The Oxford University Press. Brain: A Journal of Neurology. 2014;137:33-43.

6. WalgaardC, Lingsma $H F$, Ruts $L$ et al. Early recognition of poor prognosis in Guillin-Barre syndrome. Neurology. 2011;36(2):123-33.

7. Akbayam Sinan, Dogan Murat, AkgonCihangir et al. Clinical features and prognosis with Guillin-Barre syndrome. Annals of Indian Academy of Neurology. Official Journal of Indian Academy of Neurology. 2011;14(2):98-102.http://dx.doi. org/10.4103/0972-2327.82793;PMid:21808470 PMCid:PMC3141496

8. Sharma Bhawna, Nagpal Kadam, Sannegowda Bakki Raghavendra, Prakash Sayam. Hepatitis E with Gullain-Barre Syndrome: still a rare association. Journal of Neurovirology. 2013;19(2):186-7. http://dx.doi.org/10.1007/s13365-013-0156-z. PMid:23471727.

Cite this article : Sharma B, Paul M. Guillain—Barre Syndrome in 2016: The Centenary Advances Int. J. Med. Public Health, 2016; 6(2):111-2. 